\title{
INDICADORES DE SAÚDE AMBIENTAL: UMA ABORDAGEM ATRAVÉS DO MÉTODO PROMETHEE II
}

\author{
INDICATORS OF ENVIRONMENTAL HEALTH: AN \\ APPROACH THROUGH THE METHOD PROMETHEE //
}

\author{
José Ribamar Marques de Carvalho \\ Universidade Federal de Campina Grande - Campina Grande - PB \\ Enyedja kerlly Martins de Araújo Carvalho \\ Universidade Federal de Campina Grande - Campina Grande - PB \\ Renilson Targino Dantas \\ Universidade Federal de Campina Grande - Campina Grande - PB \\ Sérgio Murilo Santos de Araújo \\ Universidade Federal de Campina Grande - Campina Grande - PB
}

\begin{abstract}
Resumo: O grande número de fatores ambientais que podem afetar a saúde humana é um indicativo da complexidade das interações existentes e da amplitude de ações necessárias para melhorar os fatores ambientais determinantes da saúde, podendo ser variáveis sociais, econômicas e ambientais. Em face desse contexto, o presente estudo objetiva analisar a situação da performance em relação à saúde ambiental dos municípios que integram a Região do Rio Taperoá - PB.Os procedimentos metodológicos adotados neste trabalho consistiram em uma pesquisa documental e exploratória, na qual se fez uso da análise multicritério (Método PROMETHEE II). O panorama geral obtido dos municípios evidencia as fragilidades que cada ente público apresenta em relação à gestão da saúde ambiental e seus supostos desdobramentos, apontando os supostos entraves relacionados às questões sociais, econômicas e ambientais que se relacionam implícita ou explicitamente com este tipo de gestão.
\end{abstract}

Palavras-chave: Saúde Ambiental. Indicadores. Análise Multicritério.

Abstract: The large number of environmental factors that can affect human health is indicative of the complexity of existing interactions and range of actions needed to improve the environmental determinants of health factors, which may be social, economic and environmental variables. Given this context, this study analyzes the

IOs autores agradecem o apoio concedido pelo CNPq na realização da pesquisa. 
status of performance in relation to environmental health of municipalities of the Region of Rio Taperoá, PB. The methodological procedures used in this work consisted of a desk and exploratory research, which made the use of multicritério analysis (PROMETHEE Method II). The overall picture obtained from municipalities confirms the weaknesses that each public entity presents for the management of environmental health and its alleged consequences, pointing out the supposed barriers related to social, economic and environmental issues that relate implicitly or explicitly with this type of management.

Keywords: Environmental Health. Indicators. Multicriteria analysis.

\section{Introdução}

A questão ambiental ocupou os horizontes da humanidade, e vem sendo debatida em toda parte, e não é simples modismo. Ela instalouse em caráter definitivo. A razão é simples: enquanto houver a presença do homem no planeta Terra, em todo o tempo que durarem as relações ser humano-natureza, essa questão estará presente, embora num processo contínuo de mudanças e adaptações necessárias (COIMBRA, 2004).

O cenário da saúde ambiental demonstra que existe uma visão fragmentada e fragilizada dos vários atores sociais envolvidos (sociedade em geral, poder público, instituições etc.), quando, na maioria das vezes, as partes interessadas não conseguem enxergar que os problemas ambientais afetam direta ou indiretamente a saúde do ser humano. Essa deficiência demonstra a necessidade de perceber que os problemas locais, regionais, nacionais, necessitam ser revistos, repensados e discutidos como meio de buscar formas de compreender como as pessoas pensam, aprendem e agem no meio em que vivem.

O setor saúde tem sido desafiado a participar mais ativamente dos problemas relacionados à relação sociedade-natureza, seja pela atuação no cuidado das populações atingidas pelos riscos ambientais (como as intoxicações por produtos químicos, os acidentes de trânsito, as doenças transmitidas por vetores) seja pela valorização das ações de promoção e prevenção de saúde (BARCELOS; QUITÉRIO, 2006).

É nesse contexto, segundo Oenning e Carniatto (2009), que se reconhece a importância na definição e uso de indicadores de saúde ambiental como instrumento de transformação de percepções e atitudes no contexto da saúde pública. 
Segundo a Secretaria de Vigilância em Saúde do Ministério da Saúde, na Instrução Normativa 01/2005, Saúde Ambiental compreende a área da saúde pública afeita ao conhecimento científico e à formulação de políticas públicas relacionadas com a interação entre a interação, a saúde humana e os fatores do meio ambiente natural e antropogênico que a determinam, condicionam, no intuito de melhorar a qualidade de vida do ser humano sob o ponto de vista da sustentabilidade (BRASIL, 2005).

De acordo com Ribeiro (2004), o grande número de fatores ambientais que podem afetar a saúde humana é um indicativo da complexidade das interações existentes e da amplitude de ações necessárias para melhorar os fatores ambientais determinantes da saúde, podendo ser variáveis sociais, econômicas e ambientais. Nesse sentido, entende-se que um estudo que possa enfocar aspectos relacionados à saúde ambiental pode trazer contribuições interessantes ao contexto da saúde pública.

Carvalho et al. (2014) destacam que existem muitas maneiras de abordar a complexidade dos aspectos relacionados à saúde pública. Uma delas é a relação entre os seres humanos e o meio ambiente, especificamente por meio de indicadores de saúde ambiental.

Diante do exposto e dado a importância da temática em busca de um melhor entendimento da relação sociedade, saúde e meio ambiente, a análise da saúde ambiental apresenta relevância científica. Nessa perspectiva, o presente estudo procura responder ao seguinte questionamento: Qual a situação dos municípios que integram a Região do Rio Taperoá, PB, em relação à saúde ambiental?

A ideia básica está concentrada em adotar os parâmetros (indicadores) desenvolvidos por Carvalho et al. (2014) que propuseram uma metodologia baseada em indicadores de saúde ambiental e análise multicritério, considerando supostas adaptações à realidade geográfica deste estudo. Com isto objetiva analisar a situação da performance em relação à saúde ambiental dos municípios que integram a Região do Rio Taperoá, PB.

A escolha desse recorte geográfico se deu em razão de sua relevante representatividade econômica no Estado da Paraíba, uma região que contempla 23 municípios, apresenta um PIB per capita médio de $R \$ 4.602,96$, Receitas de impostos e transferências médias em torno 
de $\mathrm{R} \$ 6.150 .303,14$, um Índice de Desenvolvimento Humano Municipal - IDH-M médio baixo de 0,50 e expectativa de vida média em torno de 61 anos $^{2}$.

\section{Meio ambiente e saúde}

O ser humano é o grande agente transformador do ambiente natural e vem, pelo menos há 12 milênios, promovendo essas adaptações nas mais variadas localizações climáticas, geográficas e topográficas. O ambiente urbano é, portanto, o resultado de aglomerações localizadas em ambientes naturais transformados, e que, para a sua sobrevivência e desenvolvimento, necessitam dos recursos do ambiente natural (PHILIPPI JR. et al., 2004).

Leff (2003) coloca que aprender a aprender (a complexidade ambiental) implica uma nova compreensão do mundo que incorpora os conhecimentos e saberes arraigados em cosmologias, mitologias, ideologias, teorias e saberes práticos que estão nos alicerces da civilização moderna, no sangue de cada cultura, no rosto de cada pessoa.

Dessa forma, relacionar o meio ambiente e saúde ganha ainda mais importância, uma vez que esta relação está enraizada de aspectos inter-relacionados e que necessitam de uma visão mais ampla da realidade.

A gestão voltada para o meio ambiente e suas reflexões na saúde da população exige uma compreensão clara dos fatores econômicos, sociais, políticos, tecnológicos e ambientais que acompanharam a história do homem, possibilitando, portanto, uma reflexão sobre os diferentes modelos de desenvolvimento adotados e as direções a serem priorizadas neste terceiro milênio (PHILIPPIJR; MALHEIROS, 2005).

No Brasil, a Constituição de 1988 reflete esse quadro, com a inserção dessa questão em seu art. 225: "Todos tem direito ao meio ambiente ecologicamente equilibrado, bem de uso comum do povo e essencial à sadia qualidade de vida, impondo-se ao poder público e à coletividade o dever de defendê-lo e preservá-lo para as presentes e futuras gerações." (PHILIPPI JR, MALHEIROS, AGUIAR 2005).

\footnotetext{
2 Dados do estudo.
} 
Dessa forma, e com base nos argumentos de Borja e Moraes (2003) entende-se que o processo de construção de um sistema de indicadores de saúde ambiental envolve uma série de decisões e exige uma concepção integrada do meio ambiente no intuito de entender as conexões entre os efeitos danosos ao meio ambiente e, consequentemente, à saúde pública.

Diversos estudos mostram que as modificações ambientais provocadas pela ação antrópica vem alterando significativamente os ambientes naturais, poluindo o meio ambiente físico e consumindo recursos naturais sem critérios adequados, aumentando o risco de exposição a doenças e atuando negativamente na qualidade de vida da população (MIRANDA et al. 1994).

A avaliação de determinantes ambientais que interferem na saúde humana no âmbito das responsabilidades municipais pouco se difundiu até o presente momento no Brasil. Entretanto, a dramática mudança climática e a evolução da sociedade brasileira exigem que o acompanhamento público das ações do governo em direção às "cidades saudáveis" seja efetivamente valorizado (SOUZA et al., 2009).

Calijuri et al. (2009) enfatizam que a problemática da relação saúde-ambiente é caracterizada pela multidisciplinaridade dos fatores que a compõe. Eles podem ser de ordem política, econômica, social, cultural, psicológica, genética, biológica, física e química.

Nessa perspectiva, a problemática desse estudo configura-se entender a realidade dos munícipes a partir de um conjunto de indicadores de saúde ambiental e da análise multicritério, que permite comparar dados ambientais entre as cidades analisadas da Região do Rio Taperoá - PB, por entender que estará contribuindo para uma melhor definição e construção de políticas públicas alinhadas às necessidades locais.

\section{Estudos correlatos sobre indicadores de saúde ambiental}

A Organização Mundial da Saúde - OMS define saúde como o estado de completo bem estar físico, mental e social, e não apenas a ausência de doenças. A VIII Conferência Nacional de Saúde, realizada em 1986, propôs o entendimento do conceito de saúde como resultante das condições de alimentação, habitação, educação, renda, meio ambiente, 
trabalho, transporte, emprego, lazer, liberdade, acesso e posse da terra e acesso a serviço de saúde (ARLINDO PHILIPPI JR \& MALHEIROS 2005).

Entende-se que existem várias formas de abordar a complexidade dos aspectos relacionados à saúde pública. Uma delas é a relação entre meio ambiente e ser humano, especialmente por meio de indicadores de saúde ambiental.

A OMS define saúde pública como a ciência e a arte de promover, proteger e recuperar a saúde, por meio de medidas de alcance coletivo e de motivação da população (PHILIPPI JR. 1988). A preocupação de entender as condições sociais e ambientais e suas relações com o processo saúde doença e intervir sobre elas sempre fez parte, de diferentes modos, de diferentes momentos da história do desenvolvimento do que hoje denominamos saúde pública. (SOBRAL; FREITAS, 2010).

A problemática da relação saúde ambiente é caracterizada pela multidisciplinaridade dos fatores que a compõe. Eles podem ser de ordem política, econômica, social, cultural, psicológica, genética, biológica, física e química (CALIJURI et al., 2009)

A avaliação da saúde ambiental por meio de indicadores tem como papel principal a transformação de dados em informações relevantes para os tomadores de decisão e o público. Em particular, eles podem ajudar a simplificar um arranjo complexo de informações sobre saúde, meio ambiente e desenvolvimento, possibilitando uma visão "sintetizada" das condições e tendências existentes (VON SCHIRNDING, 2002).

Para Marzall e Almeida (2002), um indicador é uma ferramenta que permite a obtenção de informações sobre uma dada realidade e tem como principal característica a de poder sintetizar um conjunto complexo de informações, retendo apenas o significado dos aspectos analisados.

Van Bellen (2006), diz que qualquer variável e, consequentemente, qualquer indicador tem significância própria. A mais importante característica do indicador, quando comparado com os outros tipos ou formas de informações, é a sua relevância para a política e para o processo de tomada de decisão. Para ser representativo, o indicador tem que ser considerado importante tanto pelos tomadores de decisão quanto pelo público. Para ele, o objetivo dos indicadores é agregar e 
quantificar informações de um modo que sua significância fique mais aparente. Eles simplificam as informações sobre fenômenos complexos tentando melhorar com isso o processo de comunicação.

Calijuriet al. (2009) avaliaram a saúde pública da área urbana do Município de Tucuruí no Paraná, sob o ponto de vista das condições do saneamento ambiental e a composição de um sistema com menor quantidade de variáveis para discriminar áreas com distintos níveis sanitário-ambientais, e relacionar esses indicadores com a ocorrência de agravos à saúde nessas áreas.

Carvalho et al. (2014) desenvolveram uma metodologia baseada em indicadores de saúde ambiental e análise multicritério no intuito de analisar a situação da saúde em cidades, permitindo comparar dados ambientais entre municipalidades que integram a Região do Alto Curso do Rio Paraíba.

Camponogara et al. (2013) apresentaram a visão de profissionais e estudantes da área de saúde sobre a interface saúde e meio ambiente. Concluíram que o aprofundamento do debate sobre o tema no processo de formação e prática profissional em saúde é fundamental no sentido de se buscar a efetiva responsabilidade socioambiental por parte dos atores sociais atuantes no setor.

Como se nota, os resultados dos estudos supracitados adotam estratégias de uso de indicadores de saúde ambiental, identificando os fatores de risco que podem influenciar na ocorrência dos agravos da vida da população de determinado contexto geográfico específico reforçando a tese e importância que se investigar as supostas correlações existentes entre a interação ser humano-saúde-natureza.

\section{Procedimentos metodológicos}

Os procedimentos metodológicos adotados neste trabalho consistiram em uma pesquisa documental e exploratória, na qual se fez uso da análise multicritério (Método PROMETHEE II), que se configura como um método que permite obter uma ordenação final considerando os vários parâmetros de análise a partir de uma análise paritária, ou seja, compara-se um município em relação a determinado indicador e a partir disso vai obtendo comparações parciais até chegar a uma comparação final que absorve todos os indicadores e municípios. 
José R. M. Carvalho, Enyedja K. M. A. Carvalho, Renilson T. Dantas, Sérgio M. S. Araújo

Foram escolhidos os 19 indicadores de saúde ambiental (critérios) sugeridos por Carvalho et al. (2014), distribuídos em seis dimensões de maneira que fosse possível identificar aspectos do contexto geográfico estudado, conforme quadro 1. 
Quadro 1. Indicadores (critérios) e relação

\begin{tabular}{|c|c|c|}
\hline Categoria & $\begin{array}{c}\text { Indicador de Saúde Ambiental } \\
\text { (Critério) }\end{array}$ & $\begin{array}{l}\text { Relação do } \\
\text { Indicador / Fonte }\end{array}$ \\
\hline \multirow{5}{*}{ Indicadores de Saúde } & Mortalidade infantil & $\begin{array}{c}\text { Negativa - SIAB DATASUS } \\
(2009)\end{array}$ \\
\hline & $\begin{array}{c}\text { Taxa de hospitalização por } \\
\text { desidratação em menores de } 5 \\
\text { anos, por } 1000\end{array}$ & Negativa - SIAB DATSUS (2009) \\
\hline & $\begin{array}{c}\text { Taxa de mortalidade infantil por } \\
\text { diarreia (por } 1.000 \text { nascidos } \\
\text { vivos) }\end{array}$ & $\begin{array}{c}\text { Negativa - SIAB DATASUS } \\
(2009)\end{array}$ \\
\hline & $\begin{array}{c}\text { Percentual da população coberta } \\
\text { Programa Saúde da Família }\end{array}$ & Positiva - SIAB DATAUS (2009) \\
\hline & $\begin{array}{l}\text { Quantidade de estabelecimentos } \\
\text { de saúde }\end{array}$ & Positiva - IBGE (2010) \\
\hline \multirow{3}{*}{$\begin{array}{c}\text { Indicadores } \\
\text { Demográficos e } \\
\text { Sociais }\end{array}$} & Densidade demográfica & Positiva - IBGE (2010) \\
\hline & $\begin{array}{c}\text { Relação entre população urbana e } \\
\text { rural }\end{array}$ & Positiva - IBGE (2010) \\
\hline & IDH-M & Positiva - IBGE (2010) \\
\hline \multirow{3}{*}{$\begin{array}{l}\text { Indicadores } \\
\text { Econômicos }\end{array}$} & PIB per capita & Positiva - IBGE (2010) \\
\hline & $\begin{array}{c}\text { Despesa total com saúde por } \\
\text { habitante }\end{array}$ & $\begin{array}{l}\text { Positiva - SAGRES on-line } \\
\qquad(2010)\end{array}$ \\
\hline & $\begin{array}{c}\text { Transferência do SUS por } \\
\text { habitante }\end{array}$ & $\begin{array}{l}\text { Positiva - SAGRES on-line } \\
\qquad(2010)\end{array}$ \\
\hline \multirow{3}{*}{$\begin{array}{l}\text { Indicadores de coleta } \\
\text { de esgoto }\end{array}$} & $\begin{array}{c}\text { Percentual da rede sanitária via } \\
\text { esgoto }\end{array}$ & DATASUS - IBGE (2010) \\
\hline & $\begin{array}{c}\text { Percentual rede sanitária via fossa } \\
\text { séptica }\end{array}$ & Negativa - IBGE (2010) \\
\hline & $\begin{array}{l}\text { Percentual que não dispõe de } \\
\text { instalação sanitária }\end{array}$ & Negativa - IBGE (2010) \\
\hline \multirow{3}{*}{$\begin{array}{l}\text { Indicadores de coleta } \\
\text { de lixo }\end{array}$} & Percentual de lixo coletado & Positiva - IBGE (2010) \\
\hline & Percentual de lixo queimado & Negativa - IBGE (2010) \\
\hline & Percentual de lixo jogado & Negativa - IBGE (2010) \\
\hline \multirow{3}{*}{$\begin{array}{l}\text { Indicadores } \\
\text { relacionados ao } \\
\text { acesso à água e à } \\
\text { qualidade da água }\end{array}$} & $\begin{array}{c}\text { Índice de conformidade da } \\
\text { quantidade de amostras-cloro } \\
\text { residual } \%\end{array}$ & Positiva - SNIS (2009) \\
\hline & $\begin{array}{c}\text { Índice de conformidade da } \\
\text { quantidade de amostras turbidez } \\
\%\end{array}$ & Negativa - SNIS (2009) \\
\hline & $\begin{array}{c}\text { Índice de conformidade da } \\
\text { quantidade de amostras } \\
\text { coliformes totais \% }\end{array}$ & Negativa - SNIS (2009) \\
\hline
\end{tabular}

Fonte: Adaptado de Carvalho et al. 2014. 
O significado e a justificativa da escolha dos indicadores foram feitos levando em conta, primeiramente, a disponibilidade dos dados, bem como a análise da relação positivo-negativa que cada um tinha com os aspectos relacionados à saúde ambiental. Aqui foi essencial que as variáveis selecionadas para o modelo apresentassem alguma relação implícita com a problemática da relação saúde-ambiente, que é caracterizada pela multidisciplinaridade dos fatores que a compõe. Assim o modelo foi composto de 19 indicadores distribuídos em seis dimensões, conforme quadro acima.

Foram coletados dados cujas diferenças temporais fossem as menores possíveis, e ainda, que levassem em consideração a sua disponibilidade e o critério de escolha definidos.

A escolha do Método PROMETHEE II como subsídio para a elaboração da metodologia proposta nesta pesquisa se justifica em primeiro lugar, pelo seu fácil entendimento, potencializando, pois, a transparência do processo decisório e também o ordenamento das alternativas segundo os vários critérios - é simples, e os conceitos e parâmetros envolvidos em sua aplicação - indiferença, preferência fraca e preferência forte - têm um significado tangível para o decisor (JANNUZZI et al., 2009; CARVALHO et al. 2014).

Atribuiu-se pesos iguais aos indicadores, adotou-se a estratégia de que cada um dos indicadores apresenta igual peso para a análise da saúde ambiental das cidades a serem estudadas, posto que se partiu do pressuposto que nenhum indicador apresenta melhor poder de explicação em relação ao outro. Portanto, todos exerciam a mesma intensidade sobre o índice a ser proposto. $O$ mesmo raciocínio também foi adotado no estudo de Carvalho et al. (2014).

Após a coleta dos dados foram escolhidos os critérios gerais, parâmetros do estudo e a função de preferência, cuja escolha deu-se para a função do tipo 1. Nessa função, o raciocínio deve ser realizado da seguinte forma: existe indiferença entre duas alternativas a e b, somente se $f(a)=f(b)$; se as avaliações forem diferentes, há preferência estrita pela alternativa de avaliação melhor. Nesse caso, não há necessidade de definição de parâmetros. Ou seja, para o caso da pesquisa, atribuiu-se 0 se o indicador fosse indiferente ou pior do que aquele com o qual foi comparado, 1 se o indicador fosse melhor. 
Para que fosse aplicado o método PROMETHEE II, foram necessários alguns passos e procedimentos específicos. Inicialmente, foi definida uma matriz de decisão contendo $m$ alternativas (cidades) e $n$ critérios (indicadores de saúde ambiental) para as alternativas. Com os critérios definidos, definiram-se os pesos $w j$ relativos a cada um dos critérios $j$, em que $j=7, \ldots, n$.

Definidos os critérios e seus respectivos pesos, foi definido o tipo de função a ser utilizada, no caso a Função tipo 1 (Usual). Essa definição de preferência é o que a literatura define de estabelecimento de um modelo de função de preferência para cada critério $j$.

Os criadores do modelos da família PROMETHEE expuseram seis formas básicas para as funções de preferência. Essas formas e resultados podem ser expressos abaixo, na tabela 1 . 
Tabela 1. Funções de preferência - Método PROMETHEE

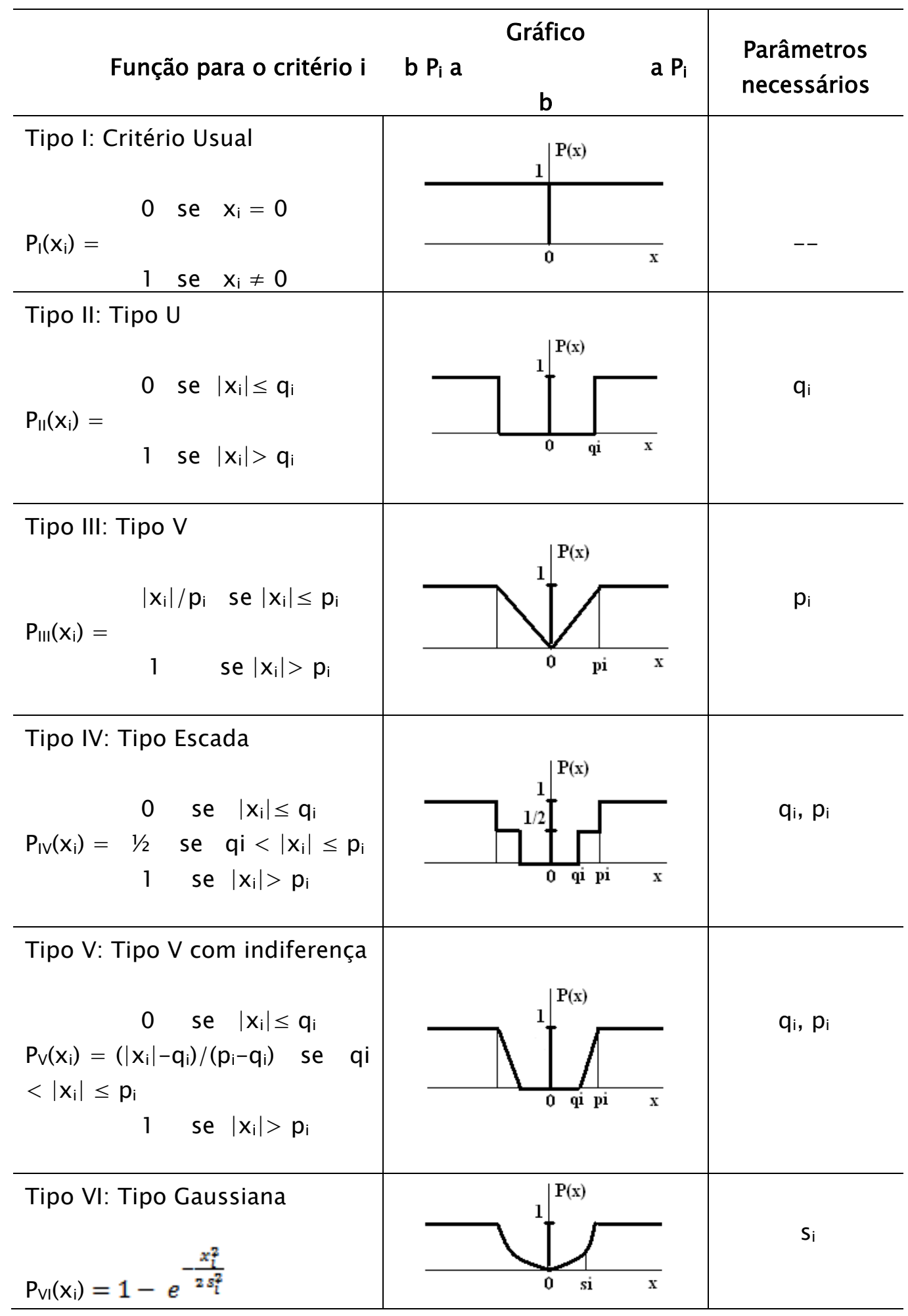

Fonte: Braga e Gobetti (2002).

Posteriormente, foram calaculados os fluxos de superação positivos $(P h i+)$ e negativos ( $P h i-)$ para cada uma das alternativas, nesse caso, representadas pela alternativa $x i$. Esses fluxos expressam 0 
quanto a cidade $x i$ supera as demais (no caso do Phit) e, no caso do Phi-, o quanto essa mesma cidade foi superada pelas outras alternativas.

Finalmente, foram obtidos os valores para $P h i+\left(\varnothing^{+}\right)$e negativos Phi- $\left(\emptyset^{-}\right)$. Foram calculados, então, os fluxos de superação líquidos ( $P h i$ ou ø) de cada uma das alternativas, que representa a diferença entre os fluxos de superação positivos e os fluxos de superação negativos. Quanto maior o Phi de uma alternativa, melhor ela se classificará na ordenação final promovida pelo modelo. Isso significa que os fluxos positivos da alternativa estão superando significativamente os fluxos negativos (BRANS, VINCKE, 1985) ${ }^{3}$.

A área de estudo foi a região da sub-bacia do Rio Taperoá - PB, uma vez que é um espaço geográfico definido segundo as características hidrológicas do Estado da Paraíba. Nessa sub-bacia, estão localizados 23 municípios: Assunção, Barra de Santa Rosa, Boa Vista, Cabaceiras, Cacimbas, Desterro, Gurjão, Juazeirinho, Junco do Seridó, Livramento, Olivedos, Parari, Pocinhos, Salgadinho, Santo André, São João do Cariri, São José dos Cordeiros, Serra Branca, Seridó, Soledade, Taperoá, Teixeira e Tenório.

Figura 1. Mapa da Paraíba com delimitação espacial e política, destacando-se os municípios com drenagem total ou parcial para a sub-bacia do Rio Taperoá

3 O software utilizado foi o Visual PROMETHEE de B. Mareshal, disponível em: http://www.promethee-gaia.net/software.html. Acesso em: out. 2014. 


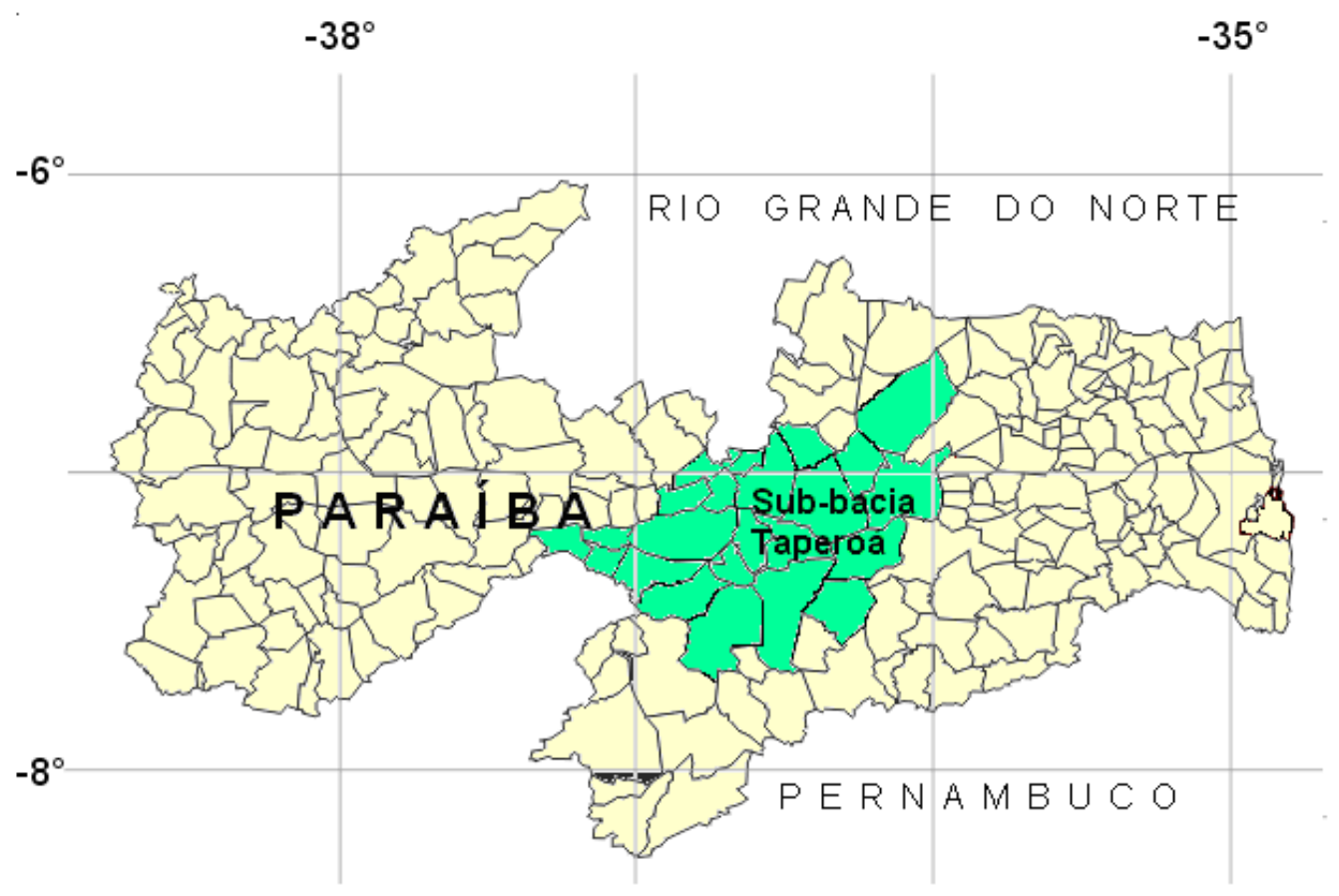

Fonte: Barreto et al. (2010).

\section{Performance dos municípios em relação à saúde ambiental}

A seguir, estão evidenciados os resultados relacionados à caracterização dos indicadores de cada uma das dimensões analisadas e consideradas no estudo. A análise foi feita de forma comparativa entre os 23 municípios analisados e que fazem parte da sub-bacia do Rio Taperoá - PB.

Após a comparação realizada pelo Método Multicritério PROMETHEE II em relação aos indicadores relacionados ao desempenho da saúde, apresentados no Quadro 1, de cada município, e após as análises positivas e negativas de cada um deles, foi possível chegar aos resultados que estão evidenciados na tabela 2 .

Observa-se que dos 23 municípios estudados, oito cidades apresentaram uma situação de maior destaque (Índice Parcial Multicritério de Saúde acima de 0,10: Soledade $(0,54)$, Teixeira $(0,25)$, Pocinhos $(0,24)$, Desterro $(0,23)$, Taperoá $(0,19)$, Salgadinho $(0,17)$ e Boa Vista $(0,11)$. Como se nota, Soledade apresentou um índice dentro dessa dimensão igual a 0,55 (demonstrando que superou no total $61 \%$ e foi superado apenas por $6 \%$ das comparações), quando se considera os indicadores mortalidade infantil, taxa de hospitalização por 
desidratação em menores de cinco anos por 1000, taxa de mortalidade infantil por diarreia (por 1.000 nascidos vivos), percentual da população coberta pelo Programa Saúde da Família e quantidade de estabelecimentos de saúde, sendo superado apenas por $6 \%$.

Como se percebe, Livramento, Juazeirinho, Serra Branca, Cacimbas, São José dos Cordeiros, Olivedos, Cabaceiras, Santo André, Junco do Seridó, Seridó e Gurjão apresentam piores desempenhos, uma vez que o Índice Multicritério foi inferior em relação aos demais municípios dessa região geográfica. Nota-se que o fluxo líquido obtido através da diferença entre os fluxos positivos $(\varnothing+)$ e os negativos ( $\varnothing-)$ apresentam valores negativos, sinalizando a necessidade de melhores estratégias da administração pública em torno dessas variáveis com vistas a proporcionar melhorias na qualidade de vida local.

O resultado relacionado às informações da saúde demonstra que os gestores locais precisam colocar na pauta de suas discussões e estratégias melhores formas de atender à população em termos de potencializar a população com a cobertura da atuação da saúde, com vistas a minimizar os casos de diarreia, casos de mortalidade infantil e maximizar a expectativa de vida. 
Tabela 2. Desempenho dos Municípios em Relação à Saúde

\begin{tabular}{lccc}
\hline Cidade & Índice & $\emptyset+$ & $\emptyset-$ \\
\hline Soledade & 0,55 & 0,61 & 0,06 \\
Teixeira & 0,25 & 0,49 & 0,24 \\
Pocinhos & 0,25 & 0,53 & 0,28 \\
Desterro & 0,23 & 0,45 & 0,22 \\
Taperoá & 0,19 & 0,50 & 0,31 \\
Salgadinho & 0,17 & 0,45 & 0,27 \\
Boa Vista & 0,11 & 0,42 & 0,31 \\
Barra de Santa Rosa & 0,05 & 0,38 & 0,34 \\
São João do Cariri & 0,02 & 0,39 & 0,37 \\
Parari & 0,02 & 0,35 & 0,33 \\
Assunção & 0,02 & 0,41 & 0,39 \\
Tenório & 0,02 & 0,36 & 0,35 \\
Gurjão & $-0,04$ & 0,33 & 0,36 \\
Seridó & $-0,05$ & 0,37 & 0,42 \\
Junco do Seridó & $-0,05$ & 0,38 & 0,43 \\
Santo André & $-0,09$ & 0,29 & 0,38 \\
Cabaceiras & $-0,15$ & 0,28 & 0,43 \\
Olivedos & $-0,15$ & 0,27 & 0,43 \\
São José dos Cordeiros & $-0,18$ & 0,30 & 0,48 \\
Cacimbas & $-0,24$ & 0,23 & 0,46 \\
Serra Branca & $-0,25$ & 0,26 & 0,52 \\
Juazeirinho & $-0,30$ & 0,35 & 0,65 \\
Livramento & $-0,37$ & 0,21 & 0,58 \\
\hline
\end{tabular}

Fonte: Dados da pesquisa, 2014.

Prosseguindo na análise, passa-se ao desempenho dos municípios quanto aos indicadores demográficos e sociais - tabela 3 (densidade demográfica, relação entre população urbana e rural, IDH-M). Gurjão se destaca, seguido de Seridó, Teixeira, Soledade, Assunção, Junco do Seridó e Desterro. Veja que naturalmente houve uma permuta de posições, obtidas pelas comparações paritárias entre os municípios versus indicadores dessa dimensão. As piores performances foram obtidas por Salgadinho, Serra Branca, São José dos Cordeiros, Cacimbas, Santo André, Barra de Santa Rosa, Olivedos, Parari, Livramento, Cabaceiras, São João do Cariri, Tenório e Juazeirinho.

Talvez uma das causas desse rendimento crítico esteja relacionada à grande ausência de políticas sociais, ou seja, os entraves sociais ainda não estão sendo sanados, sinalizando os anseios que carecem de melhorias para minimizar essas desigualdades. 
Tabela 3. Desempenho dos Municípios em Relação aos Indicadores Demográficos e Sociais

\begin{tabular}{lccc}
\hline Cidade & Índice & $\emptyset+$ & $\emptyset-$ \\
\hline Gurjão & 0,73 & 0,85 & 0,12 \\
Seridó & 0,64 & 0,82 & 0,18 \\
Teixeira & 0,64 & 0,80 & 0,17 \\
Soledade & 0,50 & 0,74 & 0,24 \\
Assunção & 0,48 & 0,73 & 0,24 \\
Junco do Seridó & 0,38 & 0,68 & 0,30 \\
Desterro & 0,18 & 0,59 & 0,41 \\
Pocinhos & 0,08 & 0,53 & 0,45 \\
Boa Vista & 0,03 & 0,52 & 0,48 \\
Taperoá & 0,00 & 0,50 & 0,50 \\
Juazeirinho & $-0,03$ & 0,47 & 0,50 \\
Tenório & $-0,03$ & 0,47 & 0,50 \\
São João do Cariri & $-0,15$ & 0,42 & 0,58 \\
Cabaceiras & $-0,15$ & 0,42 & 0,58 \\
Livramento & $-0,18$ & 0,39 & 0,58 \\
Parari & $-0,18$ & 0,41 & 0,59 \\
Olivedos & $-0,27$ & 0,35 & 0,62 \\
Barra de Santa Rosa & $-0,33$ & 0,33 & 0,67 \\
Santo André & $-0,36$ & 0,32 & 0,68 \\
Cacimbas & $-0,38$ & 0,30 & 0,68 \\
São José dos Cordeiros & $-0,39$ & 0,30 & 0,70 \\
Serra Branca & $-0,55$ & 0,23 & 0,77 \\
Salgadinho & $-0,64$ & 0,18 & 0,82 \\
\hline
\end{tabular}

Fonte: Dados da pesquisa, 2014.

De maneira análoga, passa-se ao desempenho quanto aos indicadores econômicos (tabela 4), segundo os parâmetros adotados. Parari se destaca nessa dimensão $(0,91)$, o que sinaliza certa divergência em relação às demais dimensões e indicadores analisados, visto que este município vinha sempre apresentando situações inferiores. Essa realidade leva a inferir que recursos existem, o que deve estar faltando é um melhor direcionamento para outras áreas essenciais para melhorar a realidade da saúde ambiental, o que demonstra que os anseios da sociedade podem não ser atingidos com os atuais patamares de recursos.

Como se nota, ainda não existe, nessas comunidades, uma política voltada para a geração de renda a partir das potencialidades locais, denotando um falta de política sustentável em relação a essa categoria. Soledade se apresenta em segunda melhor posição no 
ranking parcial $(0,58)$, seguido de Santo André $(0,55)$, Gurjão $(0,33)$, Boa Vista $(0,30)$, São João do Cariri $(0,21)$ e Cabaceiras $(0,15)$. Os piores desempenhos são evidenciados nos municípios de Cacimbas, Barra de Santa Rosa, Salgadinho, Livramento, Desterro, Taperoá, Junco do Seridó, Teixeira, Olivedos, Serra Branca e Seridó (já que apresentaram fluxos líquidos negativos superiores aos demais municípios). 
Tabela 4. Desempenho dos Municípios em Relação aos Indicadores Econômicos

\begin{tabular}{lccc}
\hline Cidade & Índice & $\emptyset+$ & $\emptyset-$ \\
\hline Parari & 0,91 & 0,95 & 0,05 \\
Soledade & 0,58 & 0,79 & 0,21 \\
Santo André & 0,55 & 0,77 & 0,23 \\
Gurjão & 0,33 & 0,67 & 0,33 \\
Boa Vista & 0,30 & 0,65 & 0,35 \\
São João do Cariri & 0,21 & 0,61 & 0,39 \\
Cabaceiras & 0,15 & 0,58 & 0,42 \\
Juazeirinho & 0,06 & 0,53 & 0,47 \\
Pocinhos & 0,06 & 0,53 & 0,47 \\
Tenório & 0,06 & 0,53 & 0,47 \\
Assunção & 0,03 & 0,52 & 0,48 \\
São José dos Cordeiros & 0,00 & 0,50 & 0,50 \\
Seridó & $-0,03$ & 0,48 & 0,52 \\
Serra Branca & $-0,03$ & 0,48 & 0,52 \\
Olivedos & $-0,12$ & 0,44 & 0,56 \\
Teixeira & $-0,15$ & 0,42 & 0,58 \\
Junco do Seridó & $-0,18$ & 0,41 & 0,59 \\
Taperoá & $-0,27$ & 0,36 & 0,64 \\
Desterro & $-0,33$ & 0,33 & 0,67 \\
Livramento & $-0,36$ & 0,32 & 0,68 \\
Salgadinho & $-0,39$ & 0,30 & 0,70 \\
Barra de Santa Rosa & $-0,61$ & 0,20 & 0,80 \\
Cacimbas & $-0,76$ & 0,12 & 0,88 \\
\hline Fonte: Dados & & &
\end{tabular}

Fonte: Dados da pesquisa, 2014.

Em relação à performance quanto aos resíduos sólidos (tabela 5 ) constatou-se que Seridó se destaca $(0,58)$, seguido de Boa Vista $(0,36)$, Santo André e São João do Cariri $(0,30)$, Cabaceiras $(0,24)$, Tenório $(0,21)$, Gurjão $(0,18)$. Em níveis de pouca superação do índice estão os municípios de Serra Branca, Taperoá, Soledade (necessita de melhorias nessa dimensão, já que vinha sempre apresentando anteriormente uma situação favorável em relação aos demais) e Pocinhos. Onze cidades estão em situação menos confortável em relação às demais, por apresentarem fluxo líquidos negativos. Entende-se que ainda existe ausência de uma política mais alinhada ao melhor gerenciamento dos recursos em relação às variáveis que compõem esta dimensão. 
José R. M. Carvalho, Enyedja K. M. A. Carvalho, Renilson T. Dantas, Sérgio M. S. Araújo

Tabela 5 - Desempenho dos Municípios em Relação aos Resíduos Sólidos

\begin{tabular}{lccc}
\hline Cidade & Índice & $\emptyset+$ & $\varnothing-$ \\
\hline Seridó & 0,58 & 0,79 & 0,21 \\
Boa Vista & 0,36 & 0,68 & 0,32 \\
Santo André & 0,30 & 0,65 & 0,35 \\
São João do Cariri & 0,30 & 0,65 & 0,35 \\
Cabaceiras & 0,24 & 0,62 & 0,38 \\
Tenório & 0,21 & 0,61 & 0,39 \\
Gurjão & 0,18 & 0,59 & 0,41 \\
São José dos Cordeiros & 0,12 & 0,56 & 0,44 \\
Serra Branca & 0,05 & 0,52 & 0,47 \\
Taperoá & 0,05 & 0,52 & 0,47 \\
Soledade & 0,00 & 0,50 & 0,50 \\
Pocinhos & 0,00 & 0,50 & 0,50 \\
Parari & $-0,06$ & 0,47 & 0,53 \\
Assunção & $-0,06$ & 0,47 & 0,53 \\
Barra de Santa Rosa & $-0,09$ & 0,45 & 0,55 \\
Junco do Seridó & $-0,12$ & 0,44 & 0,56 \\
Juazeirinho & $-0,18$ & 0,41 & 0,59 \\
Teixeira & $-0,24$ & 0,38 & 0,62 \\
Desterro & $-0,27$ & 0,36 & 0,64 \\
Cacimbas & $-0,27$ & 0,36 & 0,64 \\
Salgadinho & $-0,33$ & 0,33 & 0,67 \\
Livramento & $-0,36$ & 0,32 & 0,68 \\
Olivedos & $-0,39$ & 0,30 & 0,70 \\
\hline
\end{tabular}

Fonte: Dados da pesquisa, 2014.

No que se refere ao desempenho do sistema quanto ao estado qualitativo da água - gestão da água (tabela 6), foi possível observar os municípios que apresentaram os piores desempenhos (Gurjão -0,76, Serra Branca - 0,59, Barra de Santa Rosa -0,53). Os municípios de Boa Vista, Cacimbas, Desterro, Olivedos e Soledade apresentaram melhor desempenho. As cidades estudadas localizam-se em uma região semiárida e convivem com a escassez hídrica, necessitando de uma gestão mais focada em entender a complexidade que envolve a gestão da água e seus supostos efeitos à qualidade de vida da população. Espera-se que o cenário de escassez hídrica na região do estudo possa ser minimizado com a efetiva concretização do Projeto de Integração das Águas do Rio São Francisco, e que, quando de sua efetividade, a gestão pública local, regional e nacional adote mecanismos que estejam alinhados a uma gestão sustentável da água. 
Indicadores de Saúde Ambiental: uma abordagem através do Método Promethee II

Afinal, a interferência humana traz consigo consequências ao comprometimento da qualidade das águas, devido, principalmente, ao planejamento incorreto no desenvolvimento das cidades (CETESB, 2010). 
Tabela 6 - Desempenho dos Municípios em Relação à Gestão da Água

\begin{tabular}{lccc}
\hline Cidade & Índice & $\emptyset+$ & $\emptyset-$ \\
\hline Boa Vista & 0,71 & 0,74 & 0,03 \\
Cacimbas & 0,68 & 0,73 & 0,05 \\
Desterro & 0,36 & 0,56 & 0,20 \\
Olivedos & 0,33 & 0,55 & 0,21 \\
Soledade & 0,26 & 0,52 & 0,26 \\
Taperoá & 0,18 & 0,47 & 0,29 \\
Seridó & 0,17 & 0,47 & 0,30 \\
Cabaceiras & 0,14 & 0,45 & 0,32 \\
Teixeira & 0,11 & 0,44 & 0,33 \\
Junco do Seridó & 0,06 & 0,17 & 0,11 \\
Juazeirinho & 0,05 & 0,41 & 0,36 \\
Assunção & 0,00 & 0,00 & 0,00 \\
Salgadinho & 0,00 & 0,00 & 0,00 \\
Tenório & 0,00 & 0,00 & 0,00 \\
Livramento & $-0,03$ & 0,36 & 0,39 \\
Parari & $-0,06$ & 0,11 & 0,17 \\
Santo André & $-0,12$ & 0,08 & 0,20 \\
São João do Cariri & $-0,12$ & 0,32 & 0,44 \\
Pocinhos & $-0,36$ & 0,20 & 0,56 \\
São José dos Cordeiros & $-0,47$ & 0,15 & 0,62 \\
Barra de Santa Rosa & $-0,53$ & 0,12 & 0,65 \\
Serra Branca & $-0,59$ & 0,09 & 0,68 \\
Gurjão & $-0,76$ & 0,00 & 0,76 \\
\hline Fonte Dados dapesqusa
\end{tabular}

Fonte: Dados da pesquisa, 2014.

Em relação à categoria desempenho do sistema quanto ao atendimento de coleta de esgoto (tabela 7), constatou-se o seguinte desempenho para seus indicadores: Taperoá $(0,52)$, Serra Branca $(0,36)$, Assunção $(0,30)$, Santo André $(0,26)$, Junco do Seridó $(0,24)$, Soledade, $0,24)$, Barrade Santa Rosa $(0,21)$, Livramento $(0,15)$, Pocinhos $(0,09)$ e Seridó $(0,05)$. Tal resultado demonstra que os municípios localizados nessa sub-bacia hidrográfica necessitam rever algumas alternativas para melhoria em termos de coleta de esgoto, posto que essa dimensão exerce forte pressão sobre a saúde ambiental.

Muitas doenças podem ser ocasionadas pela falta de coleta adequada dos esgotos. As descargas lançadas ao meio ambiente potencializam o surgimento de vetores que causam doenças na 
população. Segundo a Agência Nacional das Águas - ANA (2010), o lançamento direto de esgoto sanitário, coletado nas redes, sem receber o devido tratamento prévio sobre os corpos d'água, pode acarretar problemas relacionados à qualidade da água e à saúde da população, por meio de fatores patogênicos que carregam consigo organismos que levam a enfermidades. 
Tabela 7. Desempenho dos Municípios em Relação à Coleta de Esgoto

\begin{tabular}{lccc}
\hline Cidade & Índice & $\emptyset+$ & $\emptyset-$ \\
\hline Taperoá & 0,52 & 0,74 & 0,23 \\
Serra Branca & 0,36 & 0,67 & 0,30 \\
Assunção & 0,30 & 0,64 & 0,33 \\
Santo André & 0,26 & 0,45 & 0,20 \\
Junco do Seridó & 0,24 & 0,61 & 0,36 \\
Soledade & 0,24 & 0,61 & 0,36 \\
Barra de Santa Rosa & 0,21 & 0,58 & 0,36 \\
Livramento & 0,15 & 0,56 & 0,41 \\
Pocinhos & 0,09 & 0,53 & 0,44 \\
Seridó & 0,05 & 0,50 & 0,45 \\
Juazeirinho & 0,03 & 0,50 & 0,47 \\
Desterro & 0,03 & 0,48 & 0,45 \\
Parari & 0,02 & 0,33 & 0,32 \\
São José dos Cordeiros & 0,00 & 0,47 & 0,47 \\
Olivedos & $-0,06$ & 0,45 & 0,52 \\
Cabaceiras & $-0,11$ & 0,42 & 0,53 \\
São João do Cariri & $-0,17$ & 0,39 & 0,56 \\
Salgadinho & $-0,18$ & 0,38 & 0,56 \\
Cacimbas & $-0,21$ & 0,36 & 0,58 \\
Boa Vista & $-0,35$ & 0,30 & 0,65 \\
Gurjão & $-0,36$ & 0,29 & 0,65 \\
Tenório & $-0,42$ & 0,27 & 0,70 \\
Teixeira & $-0,64$ & 0,17 & 0,80 \\
\hline Fonte:Dados das & & &
\end{tabular}

Fonte: Dados da pesquisa, 2014.

Os resultados obtidos com a análise encontram-se explicitados na tabela 8 , que condensa todas as dimensões estudadas. Dessa forma, a melhor posição foi obtida por Soledade $(0,37)$, seguido por Seridó $(0,20)$, Boa Vista $(0,19)$, Taperoá $(0,12)$, Assunção $(0,12)$, Parari $(0,10)$, Santo André $(0,07)$, Desterro $(0,07)$, Junco do Seridó $(0,05)$, Pocinhos $(0,04)$, Teixeira $(0,02)$, São João do Cariri $(0,02)$, Gurjão $(0,01)$ e Cabaceiras $(0,00)$. Os piores desempenhos finais foram obtidos por Livramento $(-0,21)$, seguido por Cacimbas $(-0,20)$, Barra de Santa Rosa $(-0,19)$, Salgadinho $(-0,10)$, Serra Branca $(-0,18)$, São José dos Cordeiros $(-0,16)$, Olivedos $(-0,12)$, Juazeirinho $(-0,09)$ e Tenório $(-$ $0,02)$. 
Indicadores de Saúde Ambiental: uma abordagem através do Método Promethee II

Tabela 8 - Ranking Final de Saúde Ambiental

\begin{tabular}{lccc}
\hline Cidade & Índice & $\emptyset+$ & $\varnothing-$ \\
\hline Soledade & 0,37 & 0,63 & 0,25 \\
Seridó & 0,20 & 0,55 & 0,35 \\
Boa Vista & 0,19 & 0,54 & 0,35 \\
Taperoá & 0,12 & 0,51 & 0,40 \\
Assunção & 0,12 & 0,45 & 0,34 \\
Parari & 0,10 & 0,43 & 0,33 \\
Santo André & 0,07 & 0,41 & 0,34 \\
Desterro & 0,05 & 0,46 & 0,41 \\
Junco do Seridó & 0,05 & 0,44 & 0,40 \\
Pocinhos & 0,04 & 0,48 & 0,43 \\
Teixeira & 0,02 & 0,45 & 0,43 \\
São João do Cariri & 0,02 & 0,46 & 0,44 \\
Gurjão & 0,01 & 0,44 & 0,43 \\
Cabaceiras & 0,00 & 0,45 & 0,44 \\
Tenório & $-0,02$ & 0,37 & 0,40 \\
Juazeirinho & $-0,09$ & 0,43 & 0,52 \\
Olivedos & $-0,12$ & 0,38 & 0,50 \\
São José dos Cordeiros & $-0,16$ & 0,37 & 0,53 \\
Serra Branca & $-0,18$ & 0,36 & 0,54 \\
Salgadinho & $-0,19$ & 0,29 & 0,48 \\
Barra de Santa Rosa & $-0,19$ & 0,35 & 0,54 \\
Cacimbas & $-0,20$ & 0,34 & 0,54 \\
Livramento & $-0,21$ & 0,35 & 0,56 \\
\hline Fonte: Dados da pesquisa & & &
\end{tabular}

Fonte: Dados da pesquisa, 2014.

No ranking final é possível observar a distância entre os desempenhos dos municípios, conforme retrata a figura 1 . 
José R. M. Carvalho, Enyedja K. M. A. Carvalho, Renilson T. Dantas, Sérgio M. S. Araújo

Redes (St. Cruz Sul, Online), v. 21, n² 2, p. 160 - 181, maio/ago. 2016185 
Figura 1. Rede Promethee II de performance da saúde ambiental

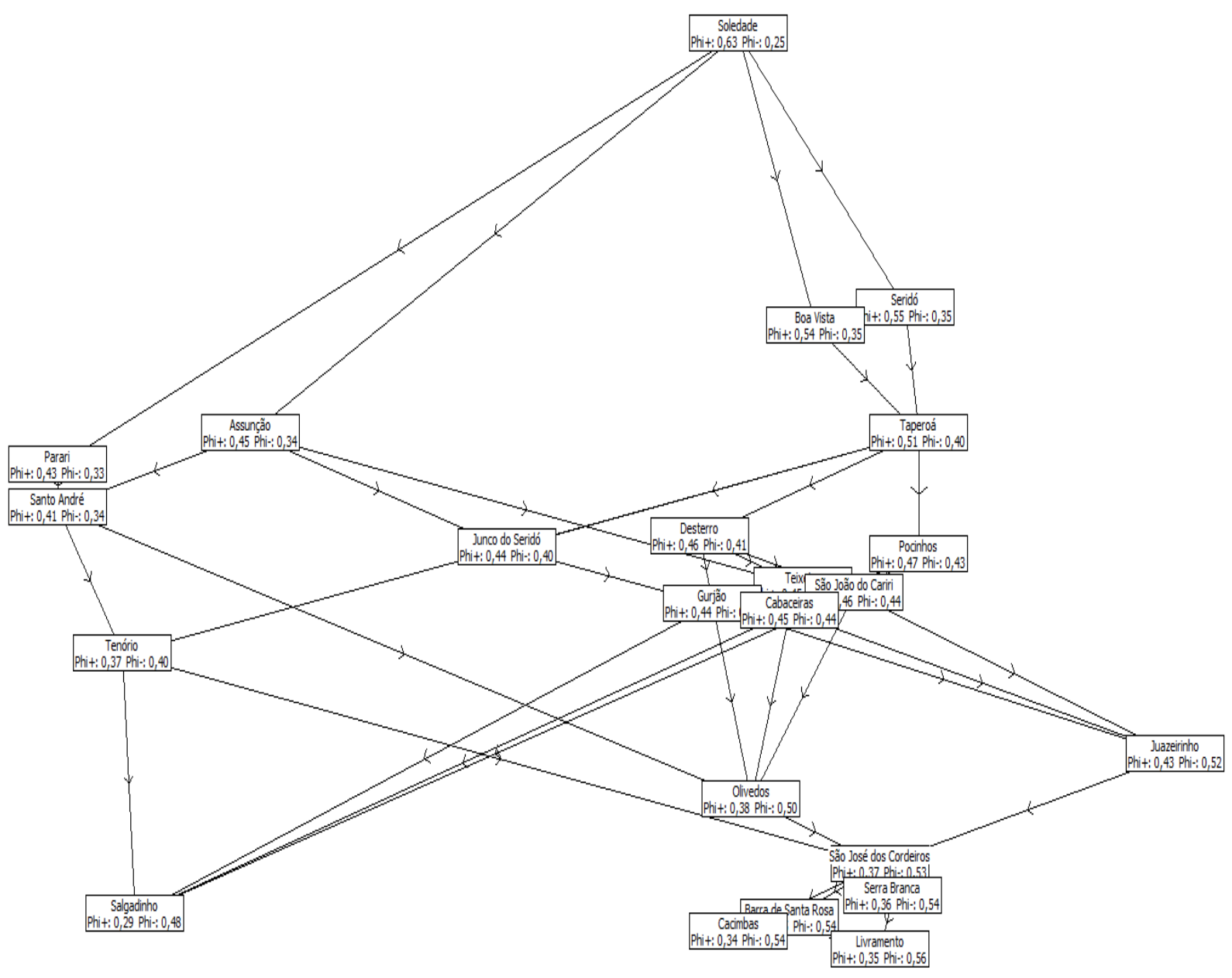

Fonte: Dados da pesquisa, 2014.

\section{Considerações finais}

O presente estudo objetivou analisar a situação da performance em relação à saúde ambiental dos municípios que integram a Região do Rio Taperoá - PB. Para tanto, fez-se o uso da pesquisa documental e exploratória e da multicritério (Método PROMETHEE II), por meio do uso de indicadores que apresentassem relação direta ou indireta com as questões que permeiam a saúde ambiental.

A realização deste estudo permitiu verificar o nível de saúde ambiental dos municípios localizados na região da sub-bacia hidrográfica do Rio Taperoá - PB, evidenciando quais aqueles que se encontram em situação menos favorável ou mais desfavorável. 
De maneira geral, pode-se afirmar que os aspectos relacionados às dimensões/categorias do estudo necessitam de mais ações com vistas a melhorias, uma vez que nelas residem entraves consideráveis relacionados à saúde ambiental da sub-bacia estudada.

Notadamente, faz-se necessário buscar alternativas que possam determinar o êxito desse cenário, sendo essencial a integração e a inclusão dos diferentes atores sociais no processo de forma que possa ser possível buscar alternativas capazes de reverter esse cenário.

Destaque-se, também, a necessidade de definir políticas públicas no sentido de estimular o crescimento da geração de alternativas sustentáveis de emprego e renda, dos níveis de educação ambiental, bem como em investimentos nas áreas diretamente relacionadas à gestão dos resíduos sólidos, saúde na tentativa de vislumbrar resultados mais satisfatórios.

Dessa forma, o panorama geral obtido dos municípios evidencia as fragilidades que cada ente público apresenta em relação à gestão da saúde ambiental e seus supostos desdobramentos, apontando os entraves relacionados às questões sociais, econômicas e ambientais que se relacionam implícita ou explicitamente com este tipo de gestão.

Há de se notar que esse cenário ainda se apresenta longe do ideal em relação à saúde ambiental, mesmo nos municípios que apresentam níveis satisfatórios em relação aos demais, como foi possível observar.

As reflexões, críticas e informações disponibilizadas neste trabalho se propõem a ampliar a discussão em torno das políticas públicas na área do estudo de modo a consubstanciar resultados mais satisfatórios a esse contexto, seja através do entendimento e ampliação da participação popular no processo de construção dessas políticas sociais, quanto para o aperfeiçoamento técnico dos órgãos gestores.

Como sugestão para trabalhos futuros, indica-se a aplicação do modelo em outros contextos específicos do Estado da Paraíba e de outros estados. Ou ainda, usar novas estratégias, como, por exemplo, a adoção de outros métodos multicritério (Analytic Hierarquic Processs; VIP Analys, ELECTRE - Elimination et Choix Traduisantla Réalité e seus desdobramentos, dentre outros), ao invés do PROMETHEE II, de modo a identificar se existem diferenças significativas entre os métodos.

\section{REFERÊNCIAS}


ANA - AGÊNCIA NACIONAL DAS ÁGUAS. Indicadores de qualidade, Indice de qualidade das águas. Disponível em: www.pnqa.ana.gov.br. Acesso em: 28 de nov. 2010.

BARRETO, J. F.; DANTAS NETO, J.; FARIAS, S. A. R. Avaliação Socioeconômica e Hídrica dos Municípios da Sub-Bacia Hidrográfica do Rio Taperoá, PB.Qualit@s Revista Eletrônica, v.9, n. 1, 2010.

BORJA, P.C.; MORAES, L.R.S. Indicadores de saúde ambiental com enfoque para a área de saneamento. Parte 1 - aspectos conceituais e metodológicos. Revista Engenharia Sanitária e Ambiental, Rio de Janeiro, v. 8, n. 1, p. 13-25, jan./mar., 2003.

BRASIL. Ministério da Saúde. Programa nacional de vigilância em saúde ambiental relacionada á qualidade da água para consumo humano. 1. Ed. Brasília: Editora do Ministério as Saúde, 2005. 72 p.

CALIJURI, M. L.; SANTIAGO, A. da F.; CAMARGO, R. de A.; MOREIRA NETO, R. F. Estudo de indicadores de saúde ambiental e de saneamento em cidade do Norte do Brasil. Eng. Sanit. Ambient. [online], vol.14, n.1, pp. 19-28, 2009.

CAMPONOGARA, S.; VIERO, C. M.; ERTHAL, G.; DIAZ, P. da S.; ROSSATO, G. C. SOARES, S. de A. PERES, R. R. Visão de profissionais e estudantes da área de saúde sobre a interface saúde e meio ambiente. Trab. educ. saúde [online], vol.11, n.1, pp. 93-111, 2013.

CARVALHO, J. R. M. de; CURI, W. F.; CARVALHO, E. K. M. A.; CURI, R. C.; CÂNDIDO, G. A. Metodologia para avaliar a saúde ambiental: uma aplicação em municípios empregando a análise multicriterial . Saude soc. [online], vol.23, n.1, pp. 204-216, 2014.

COIMBRA, J. de Á. A. Linguagem e Percepção Ambiental. In: PHILIPPI JR, Arlindo; RÓMERO, M. de A.; BRUNA, Gilda C. (Orgs.). Curso de Gestão Ambiental. Barueri, São Paulo, Manole, pp. 525-570, 2004. 
CETESB - COMPANHIA TECNOLOGIA DE SANEAMENTO AMBIENTAL. Água, Rios e Reservatórios. Disponível em: www.cetesb.sp.gov.br. Acesso em 25 mai. 2010.

FUNTOWICK, S.; MARCHI, B. de. Ciência pós-normal, complexidade reflexiva e sustentabilidade. In: LEFF, H. (Coord,) A Complexidade Ambiental. Cap. 2. Tradução Eliete Wolff. São Paulo: Cortez, 2003.

IBGE - INSTITUTO BRASILEIRO DE GEOGRAFIA E ESTATÍ́STICA. Cidades. Brasil. Disponível em: http://www.cidades.ibge.gov.br/xtras/home.php. Acesso em: 20 out. 2014.

JANNUZZI, P. M.; MIRANDA, W. L. de; SILVA, D. S. G. da. Análise multicritério e tomada de decisão em políticas públicas: aspectos metodológicos, aplicativo operacional e aplicações. Informática Pública, Belo Horizonte, ano 11, n. 1, p. 69-87, 2009.

LEFF, H. Pensar a Complexidade Ambiental. In: LEFF, H. (Coord,) $A$ Complexidade Ambiental. Cap. 1. Tradução Eliete Wolff. São Paulo: Cortez, 2003.

MALHEIROS, T. F. Indicadores Ambientais de Desenvolvimento Sustentável: um estudo de caso do uso de indicadores da qualidade do ar. Tese de Doutorado - Faculdade de Saúde Pública da USP. São Paulo, 2002.

MARZALL, K.; ALMEIDA, J. Indicadores de Sustentabilidade para Agroecossistemas: Estado da arte, limites e potencialidades de uma nova ferramenta para avaliar o desenvolvimento sustentável. Cadernos de Ciência \& Tecnologia, Brasília, v.17, n 1, jan./abr. 2000, pp.41-59.

OENNING, V.; CARNIATTO, I. Percepção Ambiental de Alunos Atingidos por Barragem em Relação a Problemas Locais. Revista Eletrônica Mestrado em Educação Ambiental, v. 23, jul.-dez., 2009.

PHILIPPI JR. A. Organizador. Saneamento do meio. São Paulo: FUNDACENTRO/USP/FSP; 1988. 
PHILIPPI JR., A.; ROMÉRO, M. de A.; BRUNA, G. C. Uma Introdução à Questão Ambiental. In: PHILIPPI JR., A.; ROMÉRO, M. de A.; BRUNA, G. C. (Orgs.). Curso de Gestão Ambiental. Barueri/SP: Manole, 2004.

PHILIPPI JR. A.; MALHEIROS, T. F. Saneamento e Saúde Pública: Integrando Homem e Ambiente. In: PHILIPPI JR. A. (Org.). Saneamento, saúde e ambiente: fundamentos para um desenvolvimento sustentável. Barueri, SP: Manole, 2005.

PHILIPPI JR. A.; MALHEIROS, T. F.; AGUIAR, A. de O. e. Indicadores de Desenvolvimento Sustentável. In: PHILIPPI JR. A. (Org.). Saneamento, saúde e ambiente: fundamentos para um desenvolvimento sustentável. Barueri, SP: Manole, 2005.

RIBEIRO, H. Saúde Publica e Meio Ambiente: evolução do conhecimento e da prática, alguns aspectos éticos. Saude\& Sociedade, v.13. n.1. jan-abr 2004. pp. 70-80.

SAGRES ON-LINE. Tribunal de Contas do Estado da Paraíba. Disponível em: https://sagres.tce.pb.gov.br/municipio_index.php. Acesso em: 15 out. 2014.

SNIS - SISTEMA NACIONAL DE INFORMAÇÕES SOBRE SANEAMENTO. Ministério das Cidades. Brasil. Disponível em: http://www.snis.gov.br/diagnostico-agua-e-esgotos. Acesso em: 10 out. 2014.

SIAB - SISTEMA DE INFORMAÇÃO DA ATENÇÃO BÁSICA. Ministério da Saúde. Brasil. Disponível em: http://datasus.saude.gov.br/sistemas-eaplicativos/epidemiologicos. Acesso em: 18 out. 2014.

SOBRAL, A.; FREITAS, C. M. de. Modelo de Organização de Indicadores para Operacionalização dos Determinantes Socioambientais da Saúde. Revista Saúde e Sociedade (online). USP, São Paulo, vol. 19, n.1. pp. 3547, 2010 
SOUZA, J. H. et al. Desenvolvimento de indicadores síntese para o desempenho ambiental. Saúde e Sociedade, São Paulo, v. 18, n. 3, p. 500-514, 2009.

VAN BELLEN, Hans Michael. Indicadores de Sustentabilidade: Uma Análise Comparativa. 2. Ed. Rio de Janeiro: FGV, 2006.

VON SCHIRNDING. Health in sustainable development planning: the role of indicators. Geneva: World Health Organization, 2002.

Submetido em 25/02/2015

Aprovado em 25/03/2016

Sobre os autores

José Ribamar Marques de Carvalho

Professor Adjunto, Universidade Federal de Campina Grande

Mestrado Profissional em Administração Pública - PROFIAP UFCG/CCJS

Centro de Ciências Jurídicas e Sociais, Unidade Acadêmica de Ciências Contábeis UFCG

E-mail: profribamar@gmail.com

Enyedja kerlly Martins de Araújo Carvalho

Mestra e Doutoranda em Recursos Naturais - PPGRN/UFCG

E-mail: enyedjakm@gmail.com

\section{Renilson Targino Dantas}

Professor do Programa de Pós-Graduação em Recursos Naturais - PPGRN/UFCG

Universidade Federal de Campina Grande, Centro de Tecnologia e Recursos Naturais, Unidade Acadêmica de Ciências Atmosféricas.

E-mail: renilson@dca.ufcg.edu.br

\section{Sérgio Murilo Santos de Araújo}

Professor do Programa de Pós-Graduação em Recursos Naturais - PPGRN/UFCG

Universidade Federal de Campina Grande, Centro de Humanidades.

E-mail: sergiomurilosa.ufcg@gmail.com 